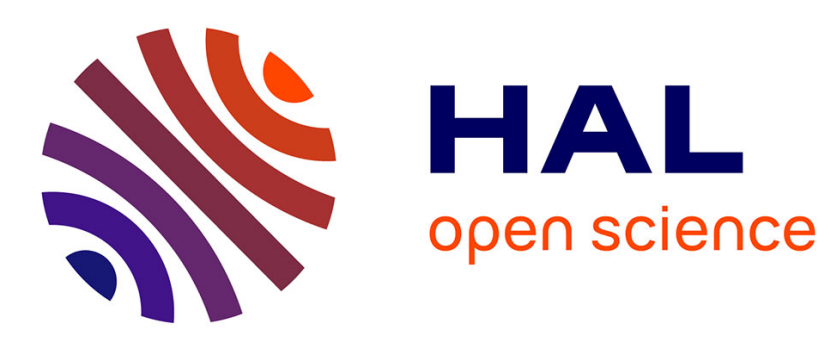

\title{
Structure-Analitycal Theory of Martensitic Unelasticity
}

\author{
V. Likhachev
}

\section{To cite this version:}

V. Likhachev. Structure-Analitycal Theory of Martensitic Unelasticity. Journal de Physique IV Proceedings, 1995, 05 (C8), pp.C8-137-C8-142. 10.1051/jp4:1995816 . jpa-00254065

\section{HAL Id: jpa-00254065 https://hal.science/jpa-00254065}

Submitted on 1 Jan 1995

HAL is a multi-disciplinary open access archive for the deposit and dissemination of scientific research documents, whether they are published or not. The documents may come from teaching and research institutions in France or abroad, or from public or private research centers.
L'archive ouverte pluridisciplinaire HAL, est destinée au dépôt et à la diffusion de documents scientifiques de niveau recherche, publiés ou non, émanant des établissements d'enseignement et de recherche français ou étrangers, des laboratoires publics ou privés. 


\title{
Structure-Analitycal Theory of Martensitic Unelasticity
}

\author{
V.A. Likhachev \\ Res. Inst. Math. Mech. (NIIMM) St.-Petersburg State Univ., Bibliotechnaya pl., 2, Petrodvorets, \\ 198904 St.-Petersburg, Russia
}

\begin{abstract}
The article presents a theory able to calculate various phenomena of martensitic unelasticity as well as of dislocation plasticity. The theory is based on the structure-analytical conception. The developed methodology accounts for such fundamental phenomena as kinetics of martensite crystals growth; influence of temperature, stress and neutron radiation; influence of nonhomogeneous strain of dislocation origin and its dependence on diffusion and radiation damage, etc. The developed theory allows to predict mechanical behaviour of crystals under an arbitrary thermomechanical and radiation effect thus making possible to solve numerous applied problems.
\end{abstract}

Introduction. Calculation of unelastic strains developing due to various processes in solids is a very complicated problem. It is not finally solved both in solid state physics and continuum mechanics. At the same time recently developed structure-analytical theory of strength $[1,2]$ presents the ground to deal with this problem. According to this methodology on the first stage micro strains corresponding to each specific process of mass transfer are calculated. Then the micro strains are summed on all orientations, physical and statistical variables with the aim of passing to the notions of continuum mechanics. On this stage, calibrating fields are added to the mean potentials and this allows to satisfy all conservation laws including those of the symmetrical character both for micro and macro levels. The described technique provides' good agreement with direct experiments [1] and this opens the perspective of solving more and more complicated problems. The present communication suggests the system of equations for calculating strains due to martensitic reactions, diffusion, radiation, elasticity, heat expansion and dislocation plasticity.

Let us agree that all the quantities refer to the crystal-physical basis $u, v, w$ of an austenite grain. We shall consider austenite to be an initial state. Then the heat expansion strain rates for austenite ${ }_{A} \dot{\beta}_{i k}^{T}$ and for martensite ${ }_{M} \dot{\beta}_{i k}^{T}$ are ${ }_{b} \dot{\beta}_{i k}^{T}=\gamma_{p q}^{b}\left(\chi_{i p} \chi_{k q} \Delta_{b M}+\delta_{i p} \delta_{k q} \Delta_{b A}\right) \dot{T}$, where $T$ is the temperature; dot means time derivative, $\chi_{i k}$ are components of the rotation matrix characterizing the orientation of a crystal physical basis relative to the parent austenite crystal. Index " $b$ " here and everywhere further denotes " $A$ " or " $\mathrm{M}$ " which means "austenite" or "martensite"; $\gamma_{\mathrm{ik}}^{\mathrm{b}}$ are the tensors of heat expansion coefficients referred to the own crystal physical bases of austenite and martensite; symbol $\Delta_{\mathrm{ik}}$ has the values $\Delta_{\mathrm{AA}}=\Delta_{\mathrm{MM}}=1$, $\Delta_{\mathrm{MA}}=\Delta_{\mathrm{AM}}=0, \delta_{\mathrm{ik}}$ is the identity tensor. 
For elastic strain rates we write ${ }_{\mathrm{b}} \dot{\beta}_{\mathrm{ik}}^{\mathrm{e}}=\left(\mathrm{C}_{\mathrm{rlts}}^{\mathrm{b}} \dot{\tau}_{\mathrm{pq}}+\frac{\partial \mathrm{C}_{\mathrm{rlts}}^{\mathrm{b}}}{\partial \mathrm{T}} \mathrm{b}_{\mathrm{pq}} \mathrm{T}\right)\left(\chi_{\mathrm{ir}} \chi_{\mathrm{k} !} \chi_{\mathrm{pt}} \chi_{\mathrm{q} s} \Delta_{\mathrm{bM}}+\delta_{\mathrm{ip}} \delta_{\mathrm{kq}} \Delta_{\mathrm{bA}}\right)$, where $\mathrm{C}_{\mathrm{ikpq}}^{\mathrm{b}}$ are elastic moduli tensors of austenite and martensite referred to their own crystal physical bases; $\tau_{\mathrm{ik}}$ is the micro stress tensor connected with the macro stress tensor in the orthogonal laboratory basis $X, Y, Z$ by the evident relation: ${ }_{b} \tau_{\mathrm{ik}}=\alpha_{\mathrm{rp}} \alpha_{\mathrm{sq}}\left(\chi_{\mathrm{ip}} \chi_{\mathrm{kq}} \Delta_{\mathrm{bM}}+\delta_{\mathrm{ip}} \delta_{\mathrm{kq}} \Delta_{\mathrm{bA}}\right) \sigma_{\mathrm{rs}}$, where $\alpha_{\mathrm{ik}}$ cosines are of the angles between the axes $\mathrm{u}, \mathrm{v}, \mathrm{w}$ and $\mathrm{X}, \mathrm{Y}, \mathrm{Z}$.

The rate of unelastic micro strain due to a direct martensitic transformation of the first order can be described by the formula $\dot{\beta}_{\mathrm{ik}}^{\Phi}=\mathrm{D}_{\mathrm{ik}} \Phi$, where $\mathrm{D}_{\mathrm{ik}}$ is the transformation strain tensor and $\Phi$ characterizes the size of the martensitic crystal.

Dislocation shears in martensite and austenite crystals also contribute to the total micro strain. Following the ideas of [1,2], we introduce an orthogonal basis $1, m, n$ of slip directing. The basis vector $\mathrm{n}$ is normal to the shear plane and allows shearing in any direction in this plane. Then we may write for the shear strain rate

${ }_{\mathrm{b}}^{\mathrm{F}} \dot{\beta}_{\mathrm{ik}}^{\mathrm{a}}=\mathrm{A}_{\mathrm{b}} \frac{{ }^{\overline{\mathrm{\tau}}^{\mathrm{r} q}}}{\mathrm{~T}_{\tau}^{\mathrm{b}}}\left(\dot{\mathrm{T}}_{\tau}^{\mathrm{b}}-{ }_{\mathrm{b}} \dot{\tau}_{\mathrm{o}}^{\mathrm{s}}\right) \mathrm{H}\left(\mathrm{T}_{\tau}^{\mathrm{b}}-\tau_{\mathrm{b}}^{\mathrm{s}}\right) \mathrm{H}\left(\dot{\mathrm{T}}_{\tau}^{\mathrm{b}}-{ }_{\mathrm{b}} \dot{\tau}_{\mathrm{o}}^{\mathrm{s}}\right)\left(\chi_{\mathrm{ip}}^{\mathrm{o}} \chi_{\mathrm{kq}}^{\mathrm{o}} \Delta_{\mathrm{bM}}+\delta_{\mathrm{ip}} \delta_{\mathrm{kq}} \Delta_{\mathrm{bA}}\right)$

Here $\quad \mathrm{b}^{\bar{\tau}_{\mathrm{ik}}^{\mathrm{r}}}={ }_{\mathrm{b}} \tau_{31}^{\prime}\left(\delta_{\mathrm{i} 1} \delta_{\mathrm{k} 3}+\delta_{\mathrm{k} 1} \delta_{\mathrm{i} 3}\right)+\tau_{32}^{\prime}\left(\delta_{\mathrm{i} 2} \delta_{\mathrm{k} 3}+\delta_{\mathrm{k} 2} \delta_{\mathrm{i} 3}\right), \quad$ and ${ }_{\mathrm{b}} \bar{\tau}_{\mathrm{ik}}^{\prime}=\alpha_{\mathrm{rp}} \alpha_{\mathrm{sq}}\left(\chi_{\mathrm{ip}}^{\mathrm{o}} \chi_{\mathrm{kq}}^{\mathrm{o}} \Delta_{\mathrm{bM}}++\delta_{\mathrm{ip}} \delta_{\mathrm{kq}} \Delta_{\mathrm{bA}}\right) \sigma_{\mathrm{rs}}^{\prime} ; \sigma_{\mathrm{ik}}^{\prime}$ is the effective stress in the place where shear takes place in austenite (when $b=A$ ) or martensite (when $b=M$ ) grains; $\chi_{i k}^{o}$ are the directing cosines between the axes of the orthogonal slip bases $(1, m, n)$ of austenite and $\left(0^{0}, m^{o}, n^{o}\right)$ of martensite; ${ }_{b} \tau_{o}^{s}$ is the crystal physical yield limit; $\tau_{b}^{s}$ is the crystal physical flow stress; $A_{b}$ is the hardening constant; $T_{\tau}^{b}=\left(\frac{1}{2} b \bar{\tau}_{i k}^{r} \quad \bar{b}_{i k}^{r}\right)^{1 / 2}$ is the intensity of shear stress acting on the slip plane; $H(x)$ is Heaviside's function. When the strain hardening is linear we have $\dot{\tau}_{\mathrm{b}}^{\mathrm{s}}={ }_{\mathrm{b}} \dot{\tau}_{\mathrm{o}}^{\mathrm{s}}+\mathrm{A}_{\mathrm{b}}^{-1} \dot{\Gamma}_{\beta}^{\mathrm{b}}$, where $\dot{\Gamma}_{\beta}^{\mathrm{b}}=\left(\frac{1}{2}{ }_{\mathrm{b}} \dot{\beta}_{\mathrm{ik}}^{\mathrm{a}} \quad{ }_{\mathrm{b}} \dot{\beta}_{\mathrm{ik}}^{\mathrm{a}}\right)^{1 / 2}$ is the shear strain intensity rate.

If we neglect aging, then it may be possible to write an evolution equation for ${ }_{b} \tau_{0}^{s}$ say in the form [1]: $\quad b_{\tau_{o}} \dot{\tau}^{s}=-\kappa_{b} \dot{T}+\alpha P_{b} T \ddot{\Gamma}_{\beta}^{b}\left(\dot{\Gamma}_{\beta}^{b}\right)^{\alpha-1}+{ }_{b} \dot{\tau}_{w}^{s}-\mathfrak{r}_{b} e^{-\left(U_{b} / k T\right)}\left(\tau_{b}^{s}-{ }_{b} \tau_{k}^{s}\right)^{m}$ 


$$
\cdot\left(1+{ }_{b} B J\right)^{\delta}\left(1+{ }_{b} B_{n} \frac{n^{b}}{n_{o}^{b}}+{ }_{b} B_{m} \frac{m^{b}}{m_{o}^{b}}\right)^{\gamma} H\left(\tau_{b}^{s}-{ }_{b} \tau_{k}^{s}\right)
$$

$b_{b} \dot{\tau}_{w}^{s}=\beta B_{w}^{b}(W)^{\beta-1} J-z_{b} e^{-\left(U_{b} / k T\right)}\left({ }_{b} \tau_{w}^{s}\right)^{\mu}\left(1+{ }_{b} B_{o} J\right)^{\delta_{o}} \cdot\left(1+{ }_{b} B_{n} \frac{n^{b}}{n_{o}^{b}}+{ }_{b} B_{m} \frac{m^{b}}{m_{o}^{b}}\right)^{\gamma / g}$, where $\kappa_{b}$, $\mathrm{P}_{\mathrm{b}}, \alpha, \mathrm{r}_{\mathrm{b}}, \mathrm{U}_{\mathrm{b}}, \mathrm{m},{ }_{\mathrm{b}} \mathrm{B}, \delta,{ }_{\mathrm{b}} \mathrm{B}_{\mathrm{n}},{ }_{\mathrm{b}} \mathrm{B}_{\mathrm{m}}, \gamma, \beta, \mathrm{B}_{\mathrm{w}}^{\mathrm{b}},{ }_{\mathrm{b}} \mathrm{B}_{\mathrm{o}}, \delta_{\mathrm{o}}, \gamma_{\mathrm{o}}$ are constants characterizing materials properties; $J$ and $W$ are the intensity and dose of radiation; $n^{b}, n_{0}^{b}$ are the actual and thermally equilibrium vacancy concentrations; $\mathrm{m}^{\mathrm{b}}, \mathrm{m}_{\mathrm{o}}^{\mathrm{b}}$ are the actual and thermally equilibrium concentrations of interstitials, $\quad b \tau_{k}^{s}$ is the equilibrium crystallographic flow stress.

Rather good approximation for $\mathbf{b}_{\mathrm{k}}^{\mathrm{s}}$ can be made in the form [3]: ${ }_{b} \tau_{k}^{s}=\tau_{F}^{b}-\kappa_{b} T+P_{b} T\left(\dot{\Gamma}_{\beta}^{b}\right)^{\alpha}+{ }_{b} \tau_{w}^{s}$, where $\tau_{F}^{b}$ is a micro stress limit distributed statistically around its mean value ${ }_{b} \tau_{F}^{o}$. The sum of $\beta_{i k}^{\Phi},{ }_{b} \beta_{i k}^{e},{ }_{b} \beta_{i k}^{T},{ }_{b}{ }_{b} \beta_{p q}^{a}$ micro strains after averaging on all orientations and other statistical variables will give the macroscopic strain tensor $\mathbf{b}^{\varepsilon_{\mathrm{ik}}}$. Tensor $b \varepsilon_{i k}$ still does not represent the total strain. One should add to it if necessary the strain produced by diffusion and the strain stimulated by radiation.

The first one can be described by the equation: ${ }_{b} \dot{\varepsilon}_{\mathrm{ik}}^{\mathrm{R}}=\mathrm{A}_{b}^{\mathrm{R}}$ Dev $\sigma_{\mathrm{ik}} \mathrm{R} \frac{\mathrm{D}_{b}^{\mathrm{R}}}{\mathrm{kT}}-{ }_{b} \mathrm{~A}_{b}^{\mathrm{R}} \dot{\mathrm{D}}_{b}^{\mathrm{R}} \delta_{i \mathrm{i}}$, where $\mathrm{R}=\mathrm{n}^{\mathrm{b}}$ for vacancies and $\mathrm{R}=\mathrm{m}^{\mathrm{b}}$ for interstitial; $\mathrm{n}^{\mathrm{b}}, \mathrm{m}^{\mathrm{b}}$ are vacancies and interstitial concentrations; $A_{b}^{R}, \quad b^{A} A_{b}^{R}$ are materials constants; $D_{b}^{R}$ is the diffusion coefficient for vacancies (when $R=n^{b}$ ) or interstitial (when $R=m^{b}$ ) in austenite (when $b=A$ ) and martensite (when $b=M$ ).

Radiation stimulated strain rate can be determined by equations ${ }_{b} \dot{\varepsilon}_{i k}^{J}={ }_{b} A_{J} \operatorname{Dev}\left(\sigma_{i k}-{ }_{b} P_{i k}^{*}\right) J$, ${ }_{b} \dot{\rho}_{i k}^{*}={ }_{b} \eta_{J} b^{\dot{\varepsilon}_{i k}}-a_{b} e^{-\left(U_{b}^{o} / k T\right)}\left[I_{2}\left({ }_{b} \rho_{i k}^{*}\right)\right]^{l}\left(1+{ }_{b} F_{J} J\right)^{g} \cdot\left(1+{ }_{b} D_{n} \frac{n^{b}}{n_{o}^{b}}+{ }_{b} D_{m} \frac{m^{b}}{m_{o}^{b}}\right)^{s} \rho_{i k}^{*}, \quad$ where ${ }_{b} A_{J}, b \eta_{J}, a_{b}, U_{b}^{o}, 1,{ }_{b} F_{J}, g,{ }_{b} D_{n},{ }_{b} D_{m}, s$ are constants, $b_{i k}^{*}$ is the tensor of effective calibrating field arising due to heterogeneous mass transfer caused by radiation effect on the crystal, $I_{2}\left({ }_{b} \rho_{i k}^{*}\right)$ is the second invariant of the tensor $b \rho_{i k}^{*}$. 
It is natural that the above relations should be complemented by point defects balance equations: $\dot{R}=D_{b}^{R} \nabla_{i} \nabla_{i} R-\left(R-R_{0}\right) / \tau_{R}^{b}+A_{j}^{b} J+A^{b} n^{b} m^{b}+A_{\varepsilon}^{b}\left({ }_{b} \dot{\varepsilon}_{i k}^{a} b_{i k}^{a}\right)^{1 / 2}$.

Here $\nabla_{i}$ is the nabla operator; $R_{0}=n_{0}^{b}$ for vacancies and $R_{0}=m_{0}^{b}$ for interstitials; $\tau_{R}^{b}$ is the life time of a point defect; $A_{j}^{b}, A^{b}, A_{\varepsilon}^{b}$ are constants; $b_{i_{i k}}^{a}$ is the macroscopic strain due to dislocation slip.

In the system presented above still undefined are variables $\sigma_{\mathrm{ik}}^{\prime}$ and $\Phi$. According to the logic of [1] potential $\sigma_{\mathrm{ik}}^{\prime}$ must take into account two factors: the effect of stress $\sigma_{\mathrm{ik}}$ and the effect of some calibrating field $\rho_{\mathrm{ik}}$, the addition of which allows to satisfy the requirements of conservation laws of the kinematical and symmetrical character both on the micro level in notions of the solid state physics and on the macro level in the notions of continuum mechanics. This can be done if we introduce the following relations: $\dot{\sigma}_{\mathrm{ik}}^{\prime}=\dot{\sigma}_{\mathrm{ik}}-{ }_{\mathrm{b}} \dot{\rho}_{\mathrm{ik}}$

$$
\begin{aligned}
{ }_{b} \dot{\rho}_{i k}= & { }_{b} \eta_{o} \quad b^{\dot{\varepsilon}_{i k}^{a}}-r_{1}^{b} e^{-\left(U_{1}^{b} / k T\right)}\left\{\left[I_{2}\left({ }_{b} \rho_{i k}\right)-{ }_{b} G\right]^{K_{b}}\left(1+C_{b} J\right)+D_{n}^{b}\left|\left(\frac{n^{b}}{n_{o}^{b}}+\frac{m^{b}}{m_{o}^{b}}-2\right)\right|^{\eta_{b}} .\right. \\
& \left.\cdot \operatorname{sign}\left(\frac{n^{b}}{n_{o}^{b}}+\frac{m^{b}}{m_{o}^{b}}-2\right)\right\} H\left[I_{2}\left({ }_{b} \rho_{i k}\right)-{ }_{b} G\right]_{b} \rho_{i k} \cdot
\end{aligned}
$$

Here ${ }_{b} \eta_{0}, r_{1}^{b}, U_{1}^{b},{ }_{b} G, K_{b}, C_{b}, D_{n}^{b}, \eta_{b}$. are constants, "sign" is the sign-function.

If we agree with the existence of the calibrating field ${ }_{b} \rho_{i k}$, we must assume that the crystal after the dislocation shear is likely to be divided into two sub-regions in one of which (where the slips are going on) the field ${ }_{b} \rho_{i k}$ is present and in the other (where there are no slips) the field - $A_{b} \rho_{i k}$ of the opposite orientation arises. Therefore one can speak of some effective tensor potential ${ }_{b} \sum \sum_{i k}^{p_{o}}=\sigma_{i k}+a_{p_{o}}^{b} b \rho_{i k}$, such that when $p_{o}$ is " $+": a_{p_{o}}^{b}=+B>0$, and when $\mathrm{p}_{\mathrm{o}}$ is " $-" \mathrm{a}_{\mathrm{p}_{\mathrm{o}}}^{\mathrm{b}}=-\mathrm{A}<0$.

This remark becomes significant at analysis of the martensite crystals growth kinetics as in accordance with the requirements of thermodynamics it is dependent on the mechanical potential that is on ${ }_{b} \sum p_{i k}$ and thus on whether $p_{o}$ is "+" or $p_{o}$ is "-".

Indeed in conformity with the Clausius - Clapeyron equation we can propose the equation for effective temperature $\dot{\mathrm{T}}_{\mathrm{p}_{\mathrm{o}}}^{*}$ which univalently specifies the state of a martensite or 
austenite crystal: $\dot{\mathrm{T}}_{\mathrm{p}_{\mathrm{o}}}^{*}=\mathrm{T}-\left(\dot{\mathrm{T}}_{\mathrm{o}} \backslash \mathrm{q}_{\mathrm{o}}\right) \alpha_{\mathrm{pi}} \alpha_{\mathrm{kq}} \mathrm{D}_{\mathrm{ik} b} \sum_{\mathrm{pq}}^{\mathrm{p}_{\mathrm{o}}}$. Here $\mathrm{T}_{\mathrm{o}}$. is the temperature of thermodynamic equilibrium, $\mathrm{q}_{\mathrm{o}}$ is the thermal effect of the reaction.

Micro strain $\beta_{\mathrm{ik}}^{\Phi}$ will have different values depending on parameter $\mathrm{p}_{\mathrm{o}}$ (" + " or "-" ) so we have to write $\dot{\beta}_{\mathrm{ik}}^{\mathrm{p}_{\mathrm{k}}}=\mathrm{D}_{\mathrm{ik}} \dot{\Phi}^{\mathrm{p}_{0}}$. Then the total martensitic unelasticity micro strain rate can be found by averaging: $\dot{\beta}_{\mathrm{ik}}^{\Phi}=(1+\mathrm{C})^{-1}\left(\dot{\beta}_{\mathrm{ik}}^{-}+C \dot{\beta}_{\mathrm{ik}}^{+}\right)$. In this case the mean martensite concentration will be $\Phi_{\Sigma}=\int_{\{s\}\{\Omega\}} d s \int_{\{\Omega} f(\Omega) \varphi(s)(1+C)^{-1}\left(\Phi^{-}+C \Phi^{+}\right) d^{3} \Omega$ and crystals with any specified value of parameter "s" appear in a quantity: $\Phi_{M}=\int_{\{\Omega\}} f(\Omega)(1+C)^{-1}\left(\Phi^{-}+C \Phi^{+}\right) d^{3} \Omega$. Let us now calculate the quantity $\Phi^{\mathrm{p}_{0}}$ Here we must say that there is a number of peculiarities in the case of the first order transformations. Firstly, one must distinguish the stages of direct and reverse transformation because of the noticeable hysteresis. Secondly, the kinetics of the reaction is determined both by temperature $T$ and stress $\sigma_{i k}$ in accordance with the effective temperature $T$. Thirdly, the transformation is characterized by the temperatures $M_{S}$ and $A_{S}$ for the start and $M_{f}$ and $A_{f}$ for the finish of direct and reverse transformations which depend on the statistical parameter " $s$ ". Fourthly, as the experiment shows, the specific crystal of martensite or austenite can not be larger than some definite size which we denote by $\Phi_{\mathrm{o}}$ and $\Phi_{\mathrm{h}}$ respectively. Fifthly, the specific crystal never grows through the entire volume of a body but is usually restricted for example to the size of a grain. At last, sixthly, if the material is subjected to radiation, large changes must take place in it according to two causes: (1) due to accumulation of radiation damages which affect the characteristic temperatures $A_{S}, M_{S}, A_{f}, M_{f}$ (they become functions of the radiation dose $W$ ), (2) due to displacement peaks formation, which causes the reverse transformation proportionally to the factor of $\Phi J$.

Considerations given above allow to write down the evolution equation for $\Phi^{\mathrm{p}_{\mathrm{o}}}$, which is valid when $A_{\mathrm{S}}-M_{\mathrm{f}} \approx A_{\mathrm{f}}-M_{\mathrm{S}}$ :

$$
\begin{aligned}
\Phi^{p_{o}} & =-\dot{T}_{p_{o}}^{*}\left\{H\left(-\dot{T}_{p_{o}}^{*}\right) H\left(1-\Phi_{M}\right) H\left(\Phi_{o}-\Phi^{p_{o}}\right) H\left[M_{s}-\Phi^{p_{o}}\left(M_{s}-M_{f}\right)-T_{p_{o}}^{*}\right]\left(M_{s}-M_{f}\right)^{-1}+\right. \\
& \left.+H\left(\dot{T}_{p_{o}}^{*}\right) H\left(\Phi_{M}\right) H\left(\Phi^{p_{0}}-\Phi_{h}\right) H\left[T_{p_{o}}^{*}+\Phi^{p_{o}}\left(A_{f}-A_{s}\right)-A_{f}\right]\left(A_{f}-A_{s}\right)^{-1}\right\}- \\
& -a J\left[\Phi^{p_{o}} H\left(\Phi^{p_{o}}\right)-\Phi_{M} \frac{M_{s}-T_{p_{o}}^{*}}{M_{s}-M_{f}} H\left(M_{s}-T_{p_{o}}^{*}\right) H\left(1-\Phi_{M}\right)\right],
\end{aligned}
$$

where $\mathbf{a}$ is a constant. 
All the above equations are nevertheless insufficient to solve arbitrary tasks. One must still take into account the rules of the material's behaviour of the austenite and martensite mixture as well as the laws of the inheriting of stress fields and strain hardening at direct and reverse reactions.

Elastic and heat expansion strains are determined by the mixture rules. Therefore $\beta_{\mathrm{ik}}^{\mathrm{y}}=\Phi^{\mathrm{p}_{\mathrm{o}}} \chi_{\mathrm{ip}} \chi_{\mathrm{kq}} \beta_{\mathrm{pq}}^{\mathrm{M}}+\left(1-\Phi^{\mathrm{p}_{\mathrm{o}}}\right) \beta_{\mathrm{ik}}^{\mathrm{A}}$,

$\beta_{\mathrm{ik}}^{\mathrm{T}}=\Phi^{\mathrm{p}_{\mathrm{o}}} \int_{0}^{\mathrm{T}} \chi_{\mathrm{ip}} \chi_{\mathrm{kq}} \gamma_{\mathrm{pq}}^{\mathrm{M}}(\mathrm{x}) \mathrm{dx}+\left(1-\Phi^{\mathrm{p}_{0}}\right) \int_{0}^{\mathrm{T}} \gamma_{\mathrm{ik}}^{\mathrm{A}} \mathrm{dx}-\chi_{\mathrm{pi}} \chi_{\mathrm{qk}} \mathrm{D}_{\mathrm{pq}}\left(1-\Phi^{\mathrm{p}_{\mathrm{o}}}\right)$

The further problem is the necessity of accounting for the inheriting of strain hardening at martensitic reactions, determined by the factor $\tau_{b}^{\mathrm{S}}-{ }_{b} \tau_{\mathrm{o}}^{\mathrm{s}}$, and of calibrating oriented stress ${ }_{b} \rho_{i k}$. Stresses ${ }_{b} \rho_{\mathrm{ik}}^{o}$ grow at the rates ${ }_{b} \dot{\rho}_{\mathrm{ik}}^{\mathrm{o}}$. Then the rate of their variation with the account of inheriting is ${ }_{M} \dot{\rho}_{i k}={ }_{M} \dot{\rho}_{i k}^{0}+{ }_{A} \dot{\rho}_{i k} \dot{\Phi}_{\Sigma},{ }_{A} \dot{\rho}_{i k}={ }_{A} \dot{\rho}_{i k}^{0}-{ }_{M} \rho_{i k} \dot{\Phi}_{\Sigma}$. It is these tensor fields ${ }_{M} \rho_{i k}$ and ${ }_{A} \rho_{i k}$ that should be taken into account when phase composition is calculated. Similarly we shall calculate strain hardening. Total strain hardening rate of austenite and martensite accounting inheritance are: $\dot{\tau}_{\mathrm{oM}}^{\mathrm{s}}=\dot{\tau}_{\mathrm{M}}^{\mathrm{s}}+\mathrm{A}_{\mathrm{A}}^{-1} \Gamma_{\beta}^{\mathrm{A}} \dot{\Phi}_{\Sigma}, \dot{\tau}_{\mathrm{oA}}^{\mathrm{s}}=\dot{\tau}_{\mathrm{A}}^{\mathrm{s}}+\mathrm{A}_{\mathrm{M}}^{-1} \Gamma_{\beta}^{\mathrm{M}} \dot{\Phi}_{\Sigma}$. Analyzing all the suggested equations one can conclude that they allow to solve a very wide scope of tasks on predicting the mechanical behaviour of crystals undergoing martensitic reactions, dislocation plasticity, diffusion and effect of radiation.

Conclusion. Suggested formalism, though the equations are somewhat cumbrous, is to the author's opinion clear in its physical contents. As the practice of its application to specific problems has shown, it can correctly describe practically all known regularities of the mechanical behaviour of alloys with thermoelastic martensitic transformation. Details one can find in [1].

\section{References}

1. Likhachev V.A., Malinin V.G. Structure-analytical theory of strength (in Russian). St.Petersburg: Nauka, 1993. - 471 P.

2. Likhachev V.A. Theory of functional mechanical properties of materials undergoing reversible martensitic transformations // Fizika Metallov i metallovedenie, 1994, vol.77, N2. P. $23-41$.

3. Volkov A.E., Likhachev V.A., Mozgunov V.F. Functional mechanical properties of materials and their computer aided design: Materials of the XXIX Interrepublican Seminar "Actual Problems of Strength", Pskov, 15 - 18 June 1993. Pskov, 1993. - P.581 591. 